\section{Evaluación de timol para el control antifúngico sobre películas de pintura}

\author{
Evaluation of thymol to antifungic \\ control on paint films
}

\author{
Bogdan Sofía ${ }^{1}$, Deyá Cecilia ${ }^{1,2}$, \\ Romagnoli Roberto ${ }^{1,2}$
}

\author{
${ }^{1}$ CIDEPINT - Centro de Investigación y Desarrollo en Tecnología de Pinturas - CIC-CONICET, Av. 52 e/ 121 y 122, La \\ Plata, B1900AYB, Bs. As., Argentina. \\ e-mail: estelectro4@cidepint.gov.ar \\ ${ }^{2}$ UNLP - Universidad Nacional de La Plata - La Plata, Bs. As., Argentina \\ e-mail: estelectro2@cidepint.gov.ar
}

\section{RESUMEN}

La colonización microbiana en el interior de los edificios preocupa en forma creciente no solo por el deterioro estético que puede ocasionar en los revestimientos sino por los problemas de salud que causan los microorganismos en personas con bajas defensas o con problemas respiratorios. En este sentido, la limpieza es fundamental para mantener las condiciones de higiene, por esto es que existen algunos productos comerciales que ayudan a la prevención y eliminación de los mohos en los revestimientos.

El timol (2-isopropil-5-metilfenol) es un monoterpeno que se encuentra como compuesto principal de varios aceites esenciales, como el de orégano y tomillo. Hay numerosos estudios que muestran el buen desempeño "in vitro" de este compuesto como antimicrobiano y desinfectante. El objetivo de esta investigación fue estudiar el desempeño del timol sobre las películas de pintura como potencial compuesto a utilizar en líquidos fungicidas para controlar la incidencia y propagación de los mohos. Para los ensayos se utilizaron paneles pintados que se colocaron sobre papel de filtro humedecido en placas de Petri. Se inocularon con una solución de esporas del hongo Alternaria alternata. Por último, se roció un grupo de paneles con una solución de timol y otro con un fungicida comercial. Como controles se utilizaron paneles rociados con el solvente del timol (etanol) y paneles sin rociar. Se incubaron a $28^{\circ} \mathrm{C}$ por 2 meses. Se observó que el spray de timol inhibió el crecimiento del hongo sobre el panel pintado mientras que el producto comercial y los controles no lo inhibieron. Por lo tanto, el uso de timol resulta una alternativa natural para la formulación de productos destinados a la prevención del desarrollo de los mohos sobre las superficies pintadas.

Palabras clave: Actividad antifúngica, Timol, Pintura, spray.

\section{ABSTRACT}

The microbial colonization is an issue of increasing concern inside buildings not only because of causing aesthetic damages in coatings but also for developing diseases in persons with low-defense systems or respiratory problems. In this sense, cleaning is fundamental to maintain the hygienic conditions and some commercial products that help to prevent and eliminate moulds in coatings have been developed.

The aim of this research was to study the performance of thymol onto paint films as a potential compound to be used in fungicide liquids to control the incidence and spread of moulds. Thymol (2-isopropyl-5methylphenol) is a monoterpene found as the main compound in various essential oils, such as thyme and oregano. Numerous "in vitro" studies have shown the good performance of this compound as an antimicrobial and disinfectant. Painted panels were placed on moistened filter paper in Petri and, a spore solution of $\mathrm{Al}$ ternaria alternata, an environmental fungus was inoculated onto the paint. Finally, a set of panels was sprayed with a solution of thymol and another set with a commercial product. Sprayed panels with the thymol solvent (ethanol) and not sprayed panels were used as controls. Petri dishes were incubated at $28^{\circ} \mathrm{C}$ for 2 months. It was observed that the thymol spray inhibited the fungus growth onto painted panels, while the commercial product and the controls did not show inhibition. Therefore, thymol is a natural alternative to 
formulate products that help to prevent the development of moulds on painted surfaces.

Keywords: Antifungal activity, Thymol, Coating, spray.

\section{INTRODUCCIÓN}

Las pinturas de base acuosa son aquellas en las que el vehículo es una emulsión de la resina en agua. Al ser aplicadas, la emulsión se rompe al eliminarse el agua por secado, obteniéndose así una fase oleosa continua. En las formulaciones de este tipo de pinturas se han quitado los disolventes orgánicos -VOCs- reduciéndose así la emisión de estos compuestos y, por ende, la contaminación ambiental. La desventaja de estas pinturas es el gran contenido de aditivos que requieren para su formulación: espesantes (en su mayoría a base de celulosa), emulsionantes, plastificantes, coloides, entre otros. Todos ellos susceptibles de biodeterioro [1- $\underline{3}]$. La colonización microbiana en el interior de los edificios preocupa en forma creciente desde las últimas décadas, no solo por los deterioros estéticos que pueden ocasionar en los revestimientos sino por los problemas de salud que causan los microorganismos en personas con bajas defensas o con problemas respiratorios. Existe mucha bibliografía que relaciona los problemas de salud con la contaminación fúngica en ambientes cerrados.

Los hongos son un grupo muy grande, diverso y heterogéneo de organismos que se encuentran en prácticamente todos los nichos ecológicos. Entre los géneros más comúnmente aislados en el interior de los edificios se encuentran Penicillium, Aspergillus, Cladosporium y Alternaria [4-7].

La limpieza es fundamental para mantener las condiciones de higiene, existiendo una creciente necesidad de priorizar la limpieza a nivel de la superficie, por ejemplo, para la prevención y control de las infecciones en hospitales, especialmente porque los patógenos son cada vez más resistentes a los agentes antimicrobianos [ $\underline{8}-\underline{10}]$. Por esto, existen en el mercado algunos productos comerciales que ayudan a la prevención y eliminación de los mohos en los revestimientos.

El timol (2-isopropil-5-metilfenol) es un monoterpeno fenólico que se encuentra como componente de varios aceites esenciales, principalmente de orégano, tomillo y ajedrea. Utilizado ya en el antiguo Egipto para la preservación de las momias, este compuesto se encuentra como parte de la formulación de diversos medicamentos, pastas dentales, entre otros productos. Hay numerosos estudios que muestran la eficacia de este compuesto como bactericida, fungicida, insecticida, nematicida, varroocida y desinfectante en general [1113].

El objetivo de esta investigación fue estudiar, de manera preliminar, el desempeño del timol aplicado sobre las películas de pintura como potencial compuesto a utilizar en líquidos fungicidas para controlar la incidencia y propagación de los mohos.

\section{MATERIALES Y MÉTODOS}

\subsection{Compuestos y Cepas}

Se utilizó Timol, calidad analítica, provisto por Sigma-Aldrich. Para los ensayos se preparó una solución de timol en etanol $96 \%$, con una concentración final de $1 \mathrm{mg} / \mathrm{ml}$. También se utilizó un producto comercial promocionado como eliminador de moho. Se preparó una dilución 1/10 en agua destilada, según recomendación del fabricante.

Dado que el producto comercial no especifica composición, se realizaron espectros de Infrarrojo con Transformada de Fourier (FTIR) de ambos compuestos para descartar la posibilidad de que el producto comercial contenga timol. Para realizar las medidas se usó la técnica de Reflexión Total Atenuada (ATR-FTIR) con una ventana de $\mathrm{ZnSe}$ en un espectrofotómetro Perkin-Elmer Spectrum One.

El aislamiento fúngico utilizado en este ensayo, Alternaria alternata, fue previamente obtenidos de películas de pinturas contaminadas. La suspensión de esporas fue preparada a partir de subcultivos en placa del hongo con un crecimiento de 25 días a $25^{\circ} \mathrm{C}$ en medio de cultivo agarizado (MCA: 1,5g agar-agar, $1 \mathrm{~g}$ dextrosa, 0,5 g proteasa peptona, $0,1 \mathrm{~g} \mathrm{KH}_{2} \mathrm{PO}_{4}, 0,05 \mathrm{~g} \mathrm{MgSO}_{4}, \mathrm{H}_{2} \mathrm{O}$ destilada hasta completar $100 \mathrm{ml}$.). Las esporas fueron removidas de las placas y depositadas en frascos con $5 \mathrm{ml}$ de una solución de $\mathrm{NaCl} 0,85 \%$ p/v y Tween-20 $0,005 \% \mathrm{p} / \mathrm{v}$. La concentración de esporas fue ajustada a $10^{6}$ esporas/ml utilizando la cámara de Neubauer. 


\subsection{Formulación y elaboración de la pintura}

Se formuló una pintura acrílica al agua, sin ningún tipo de biocida, cuya composición se presenta en la Tabla 1. Se elaboró en una dispersora de alta velocidad y se conservó en un frasco cerrado a temperatura ambiente hasta su uso.

Tabla 1: Composición de la pintura.

\begin{tabular}{l|l|l}
\hline COMPONENTE & $\%(\mathbf{P} / \mathbf{P})$ & COMENTARIO \\
\hline Agua & 25,2 & Solvente \\
\hline Antiespuma & 0,3 & \\
\hline Espesante & 0,5 & Celulósico \\
\hline Dispersantes & 0,45 & \\
\hline Humectante & 0,15 & \\
\hline $\mathrm{TiO}_{2}$ & 19,0 & Pigmento opacante \\
\hline $\mathrm{CaCO}_{3}$ natural & 41,6 & Pigmento carga \\
\hline $\mathrm{CaCO}_{3}$ precipitado & 3,7 & Pigmento extendedor \\
\hline Resina acrilica estirenada & 7,2 & \\
\hline Aguarrás & 1,3 & Coalescente insoluble \\
\hline Butilglicol & 0,6 & Coalescente soluble \\
\hline
\end{tabular}

\subsection{Evaluación del crecimiento fúngico sobre paneles pintados}

La eficacia del timol contra el hongo Alternaria alternata se evaluó sobre paneles de vidrio de 2,5 cm x 2,5 $\mathrm{cm}$, recubiertos con dos manos de pintura. Una vez aplicada la pintura se dejaron secar los paneles en ambiente de laboratorio por 15 días (curado de la pintura). Pasado ese tiempo, se esterilizaron con luz UV, 20 minutos de cada lado y, luego, se colocaron en placas de Petri conteniendo papel de filtro con $1 \mathrm{ml}$ de agua estéril, para generar una atmósfera húmeda. Se inoculó cada panel de pintura con $100 \mu 1$ de una suspensión de esporas $\left(10^{6}\right.$ esporas/ml solución fisiológica) del hongo ensayado. Por último, se procedió a realizar el spray sobre los paneles: un grupo de paneles se roció con $2 \mathrm{ml}$ de una solución de timol $(1 \mathrm{mg} / \mathrm{ml})$, otro con el producto comercial en dilución 1/10, según recomendación del fabricante y otro con el producto comercial concentrado. Como controles se utilizaron paneles rociados con el solvente del timol (etanol) y paneles sin rociar. Se incubaron a $28^{\circ} \mathrm{C}$ por 2 meses.

El crecimiento del hongo fue estimado como porcentaje de cobertura sobre la superficie, por medio de inspección visual, a simple vista y en lupa. Los paneles se calificaron según se muestra en la tabla 2.

Tabla 2: Calificación del crecimiento fúngico en placa

\begin{tabular}{ll}
\hline OBSERVACIÓN DEL CRECIMIENTO & CALIFICACIÓN \\
\hline Sin crecimiento & 0 \\
\hline Crecimiento en trazas $(<10 \%)$ & 1 \\
\hline Crecimiento bajo $(10-30 \%)$ & 2 \\
\hline Crecimiento moderado $(30-70 \%)$ & 3 \\
\hline Crecimiento alto (70\% hasta cobertura completa) & 4 \\
\hline
\end{tabular}

\section{RESULTADOS}

\subsection{Espectros FTIR}

Los espectros obtenidos por ATR-FTIR (figura 1) muestran que el compuesto comercial no contiene timol en su composición dado que los picos característicos de este último no están presentes en el espectro del producto comercial. 


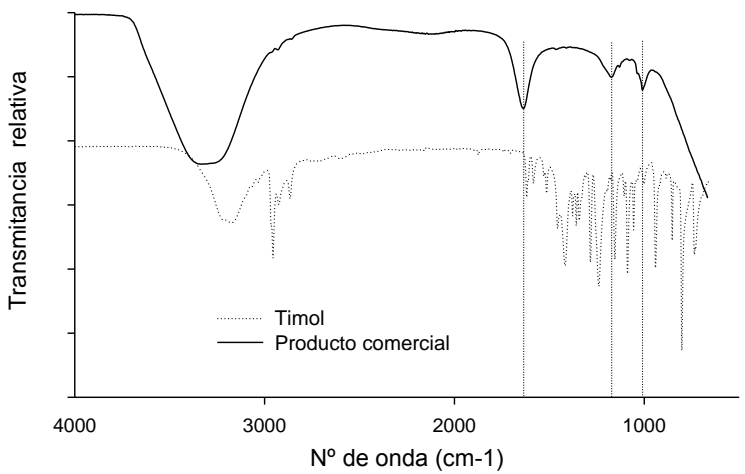

Figura 1: FTIR del timol y del producto comercial.

\subsection{Evaluación del crecimiento fúngico sobre paneles pintados}

Los resultados obtenidos para el ensayo de los paneles pintados, se resumen en la tabla 3 y las fotografías de los paneles se muestra en la figura 2. El control sin spray muestra la máxima calificación, es decir 4, que corresponde a un porcentaje de cobertura mayor al 70\%, mientras que el control con solvente (etanol) se lo calificó con 3 puntos. Estos resultados muestran que el solvente también ejerce cierta influencia sobre el crecimiento fúngico, que puede resultar beneficioso en una formulación para un potencial producto comercial, como una barrera más de protección. En cuanto al desempeño del producto comercial, se puede observar que utilizándolo de manera concentrada produce un efecto contrario al deseado, pues favorece el crecimiento del hongo, de hecho obtuvo la máxima calificación. Comparativamente, con el control sin spray, si bien obtuvieron la misma calificación, se ve que el crecimiento no es tan agresivo como en este último. Por otro lado la dilución recomendada por el fabricante logró disminuir el crecimiento respecto del control, pero solo en parte quedando prácticamente igual que el control con solvente. Finalmente, el timol muestra una muy buena inhibición del crecimiento fúngico luego de dos meses de ensayo, dado que el desarrollo del hongo fue mínimo.

Los resultados aquí obtenidos concuerdan con estudios previos realizados in vitro sobre el desempeño del timol como antifúngico sobre un amplio espectro de hongos [13-15], en particular PERINA et al. [15], reportan la activad de dicho compuesto contra A. alternata con resultados muy favorables. Incluso se ha reportado la utilización del timol en conservación del papel [11], en plásticos para conservar alimentos [16-18], y otros usos más conocidos, como productos de higiene bucal y fármacos [12]. Pero, hasta el momento, no se ha encontrado bibliografía sobre la utilización del timol en pinturas como sustrato.
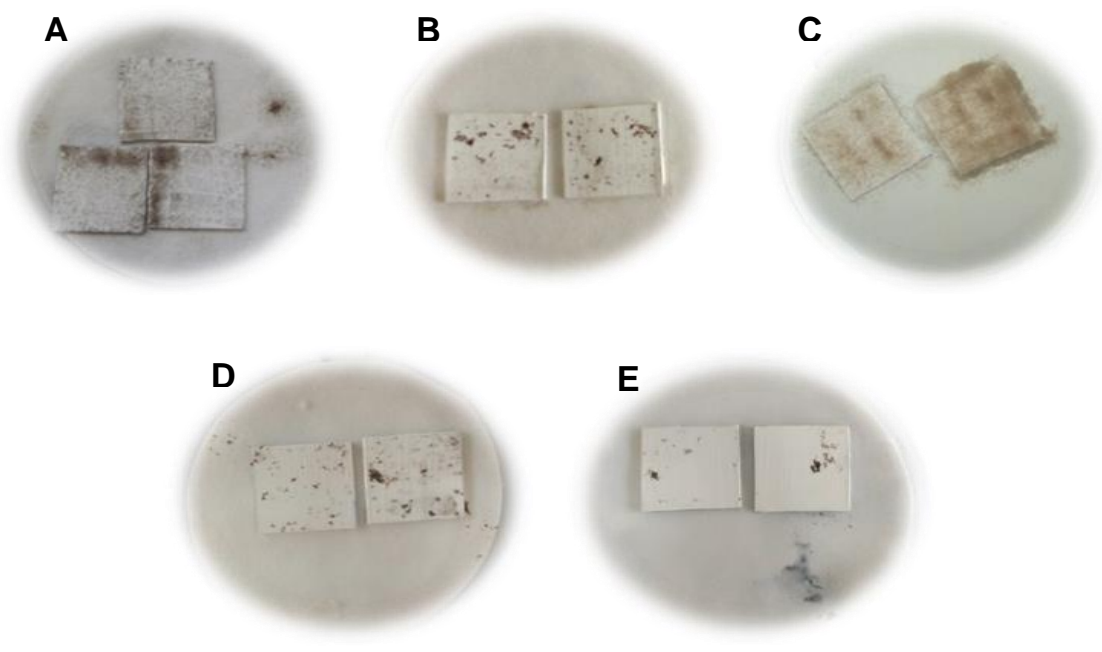

Figura 2: Crecimiento fúngico sobre paneles: (A) Control sin spray; (B) Control solvente; (C) Spray producto comercial concentrado; (D) Spray producto comercial dil. 1/10; (E) Spray timol 1mg/ml. 
Tabla 3: Crecimiento fúngico sobre paneles pintados

\begin{tabular}{ll}
\hline & CALIFICACIÓN \\
\hline Control sin spray & 4 \\
Control solvente (etanol) & 3 \\
Spray producto comercial concentrado & 4 \\
Spray producto comercial dilución 1/10 & 3 \\
Spray timol & 1 \\
\hline
\end{tabular}

Las fotografías de la figura 3 muestran la observación en lupa de los paneles rociados con timol y con el solvente. Las figuras 3-A y 3-B muestran abundante desarrollo de micelio y esporas, cubriendo prácticamente toda la superficie de la pintura, con algunas zonas más densas que otras. En la figura 3-C (timol) se observa claramente que el hongo tiene un desarrollo de micelio mucho menor; los que han podido crecer están aislados y son de tamaño reducido en comparación con el control. Esto esta de acuerdo a lo encontrado en bibliografía donde se reporta que el timol actúa sobre los hongos a nivel de las membranas, afectando su permeabilidad, y alterando la morfología de las hifas, que conlleva a una reducción en el diámetro de las hifas e incluso a la ruptura de las paredes de las hifas [11, $\underline{19}-\underline{20}]$

A)

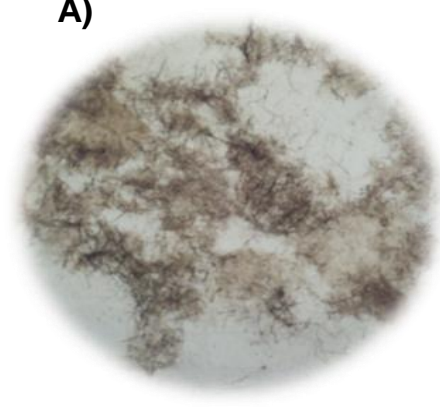

B)

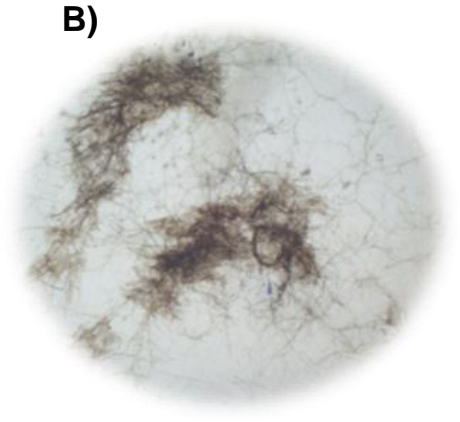

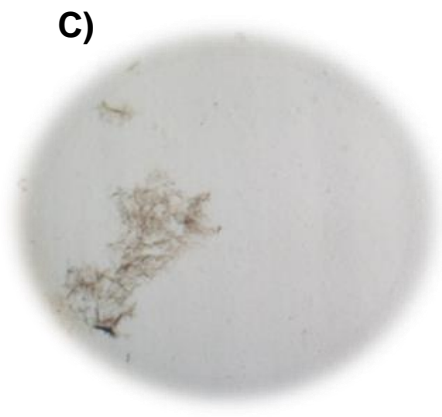

Figura 3: Observación en lupa paneles (A) Control solvente (etanol); (B) Spray producto comercial; (C) Spray timol 1 $\mathrm{mg} / \mathrm{ml}$.

\section{CONCLUSIONES}

Se observó que el spray de timol inhibió el crecimiento del hongo sobre el panel pintado mientras que el producto comercial y los controles no lo inhibieron. Por lo tanto, el uso de timol resulta una alternativa natural para la formulación de productos destinados a la prevención del desarrollo de los mohos sobre las superficies pintadas.

Este tipo de propuesta resulta atractiva y con buen potencial, pero es necesario seguir investigando y trabajar en conjunto con empresas, para hacer viable la selección de ideas, tecnologías y compuestos "ecoamigables" para la generación de productos comerciales.

\section{AGRADECIMIENTOS}

Al CONICET, la CICBA y la UNLP por el apoyo recibido. Al Dr. Guillermo Blustein por su colaboración. A la empresa Diransa por la provisión de resina y aditivos para la formulación de la pintura.

\section{BIBLIOGRAFÍA}

[1] GUIAMET P.S., VIDELA H.A., "Microbiological Spoilage of Aqueous Based Surface Coatings", Corrosion Reviews, v. 14, n 1-2, pp. 47-58, 1996. 
[2] RHOAdES A. M., DOUGLAS A. W., BRUHASPATHY M., et al., "Interactions of an antimicrobial peptide (Ac-RRWWRF-NH2) and surfactants: Towards antimicrobial peptide additives for coatings applications", Progress in Organic Coatigs, v. 58, pp. 209-216, 2007.

[3] BRAVERY A.F, "Biodeterioration of Paint-a State-of-the-Art Comment", In: HOUGHTON D. R., SMITH R. N., EGGINS H. O. W. (eds), Biodeterioration 7, 1 ed., chapter 15, Netherlands, Elsevier Science Publishers Ltd, 1988.

[4] LI, D., YANG, C. S., "Fungal contamination as a major contributor to sick building syndrome", Advance in Applied Microbiology, v. 55, pp. 31-112, 2004.

[5] KHAN, H., KARUPPAYIL, M., "Fungal pollution of indoor environments and its management", Saudi Journal of Biological Sciences, v. 19, pp. 405-426, 2012.

[6] NEGRIN, M., DEL PANNO, M.T., RONCO, A.E., "Study of bioaerosols and site influence in the La Plata area (Argentina) using conventional and DNA (fingerprint) based methods", Aerobiologia, v. 23, pp. 249-258, Dic. 2007.

[7] ALEXOPOULOS, C. J., MIMS, C. W., BLACKWELL, M., Introductory Mycology, 4th ed., New York, John Wiley and Sons, Inc., 1996.

[8] MAILLARD, J., "Antimicrobial biocides in the healthcare environment: efficacy, usage, policies, and perceived problems", Therapeutics and Clinical Risk Management, v. 1, n 4, pp. 307-320, Ene. 2005.

[9] DANCER, S. J., "Hospital cleaning in the 21st century", European Journal of Clinical Microbiology \& Infectious Diseases, v. 30, n 12, pp. 1473-1481, Dic. 2011.

[10] DANCER, S. J, "Cleaning and decontamination of the healthcare environment", In: WALKER J. H (eds), Decontamination in Hospitals and Healthcare, 1 ed., chapter 15, Cambridge, UK, Woodhead Publishing Limited, 2014.

[11] SEQUEIRA, S., CABRITA, E. J., MACEDO, M. F., "Antifungals on paper conservation: An overview", International Biodeterioration \& Biodegradation, v. 74, pp. 67-86, 2012.

[12] BURT, S., "Essential oils: their antibacterial properties and potential applications in foods-a review", International Journal of Food Microbiology, v. 94, pp. 223-253, 2004.

[13] MAREI, G., ABDEL RASOUL, M. A., ABDELGALEIL, S. A. M., "Comparative antifungal activities and biochemical effects of monoterpenes on plant pathogenic fungi", Pesticide Biochemistry and Physiology, v. 103, pp. 56-61, 2012.

[14] ZABKA, M., PAVELA, R., "Antifungal efficacy of some natural phenolic compounds against significant pathogenic and toxinogenic filamentous fungi”, Chemosphere, v.93, n 6, pp. 1051-1056, Oct. 2013.

[15] PERINA, F. J., AMARAL, D. C., FERNANDES, R. S., et al., "Thymus vulgaris essential oil and thymol against Alternaria alternata (Fr.) Keissler: effects on growth, viability, early infection and cellular mode of action", Pest. Manag. Sci., 2014. doi: 10.1002/ps.3933

[16] GUARDA, A., RUBILAR J.F., MILTZ J., et al., "The antimicrobial activity of microencapsulated thymol and carvacrol", International Journal of Food Microbiology, v.146, n 2, pp.144-150, mar. 2011.

[17] PILATI, F., DEGLI ESPOSITO, M., BONDI, M., et al., "Designing of antibacterial plastics: thymol release", Journal of Materials science, v 48, n 12, pp. 4378-4386, Jun. 2013.

[18] DEGLI ESPOSITO, M., PILATI, F., BONDI, M., et al., "Preparation, characterization, and antibacterial activity of photocured thymol-doped acrylic resins", Journal of Coatings Technology and Research, v10, n3, pp. 371-379, Mayo 2013.

[19] CHAVAN P. S., TUPE S. G., "Antifungal activity and mechanism of action of carvacrol and thymol against vineyard and wine spoilage yeasts", Food Control, v.46, pp. 115-120, Dic. 2014.

[20] ŜEGVIĆ, K.. M., KOSALEC I., MASTELIĆ J., PIECKOVÁ E., PEPELJNAK S., “Antifungal activity of thyme (Thymus vulgaris L.) essential oil and thymol against moulds from damp dwellings", Letters in Applied Microbiology, v 44, pp. 36-42, 2007. 